\title{
Improved Vlasov Antenna with Curved Cuts and Optimized Reflector Position and Shape
}

\author{
H. M. El Misilmani, ${ }^{1}$ M. Al-Husseini, ${ }^{2}$ and K. Y. Kabalan ${ }^{1}$ \\ ${ }^{1}$ ECE Department, American University of Beirut, P.O. Box 11-0236, Beirut 1107 2020, Lebanon \\ ${ }^{2}$ Beirut Research and Innovation Center, Lebanese Center for Studies and Research, Beirut 2030 8303, Lebanon \\ Correspondence should be addressed to H. M. El Misilmani; hilal.elmisilmani@ieee.org
}

Received 24 October 2014; Accepted 18 January 2015

Academic Editor: Jun $\mathrm{Hu}$

Copyright (C) 2015 H. M. El Misilmani et al. This is an open access article distributed under the Creative Commons Attribution License, which permits unrestricted use, distribution, and reproduction in any medium, provided the original work is properly cited.

\begin{abstract}
This paper presents a Vlasov antenna with curved cut shape and improved reflector position and geometry suitable for high power microwave applications. The curved shape of the proposed cut totally eliminates the sharp edges and angles present in Vlasov antennas with step and bevel cuts. Furthermore, with the proposed reflector configuration, the wave is radiated in the direction of the axis of the waveguide. A Vlasov antenna, designed for operation at $3 \mathrm{GHz}$, is used to compare the three cut types. An additional comparison is conducted to validate the concept of the enhanced reflector position, using the bevel-cut antenna and the improved cut. The proposed antenna results in increased antenna gain and in good performance in terms of sidelobe level and half-power beamwidth, with maximum radiation directed toward the axis of the waveguide center.
\end{abstract}

\section{Introduction}

High power microwave (HPM) sources, such as the backward-wave oscillator (BWO), the gyrotron, and the vircator (virtual cathode oscillator), generate power in cylindrically symmetric transverse electric $\mathrm{TE}_{0 n}$ or transverse magnetic $\mathrm{TM}_{0 n}$ modes. The sidelobe generation, gain reduction, and inefficient power loading on the antenna aperture make these modes unsuitable for driving conventional antennas. This gave the idea of using mode converters at the output of these sources to convert these modes into a plane-parallel linearly polarized beam.

A Vlasov antenna [1-3], which is one of the most known mode converters used, is composed of a cylindrical waveguide with a shaped end, which can directly radiate energy from cylindrically symmetric modes in circular waveguides, without the need for an additional mode converter. The two well-known Vlasov antenna types come with a step cut and with a beveled cut [4]. The first type, a waveguide aperture with a step cut, originally suggested by Vlasov, has sharp edges and therefore may suffer from electrical breakdown when radiating HPM [5]. The beveled cut was suggested by
Wada and Nakajima [6] to avoid the sharp points of the step cut, leading to a more suitable shape for usage in HPM applications.

A comparison of the performance of bevel-cut and stepcut Vlasov antennas in HPM is conducted by Ruth et al. in [7], concluding that the bevel cut has better performance in such applications. A series of Vlasov-type high power microwave launchers have been investigated with several slant-cut angles in [8]. Additional experimental results and theoretical investigation of $\mathrm{TE}_{0 n}$ and $\mathrm{TM}_{0 n}$ mode Vlasov launchers have been presented in $[9,10]$. Other studies focused on increasing the gain of bevel-cut and step-cut Vlasov antennas. Dahlstrom et al. added a reflector to a bevel-cut Vlasov antenna to increase its gain and better direct the main beam [11]. Fazaelifar and Fatorehchy proposed in [12] two methods for increasing the gain of a bevel-cut antenna, one using a parabolic cylinder reflector and the second using a horn. Moreover, Zhang et al. studied the step cut in the presence of a parabolic reflector [13]. Other work discussed the use of dualreflector in the presence of Vlasov antennas [14,15]. Improved beam focusing of high powered microwaves radiating from a Vlasov antenna has been also achieved by applying a flare to 


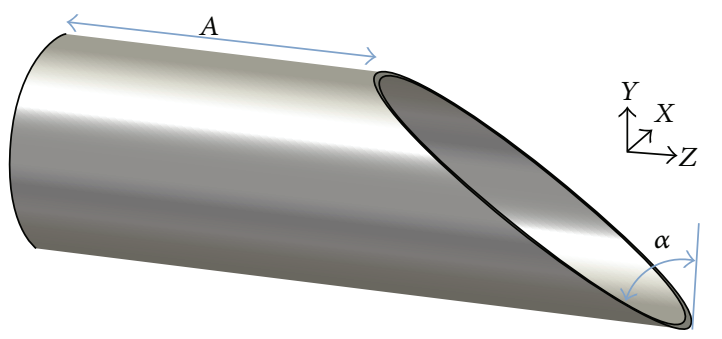

(a) Bevel-cut design

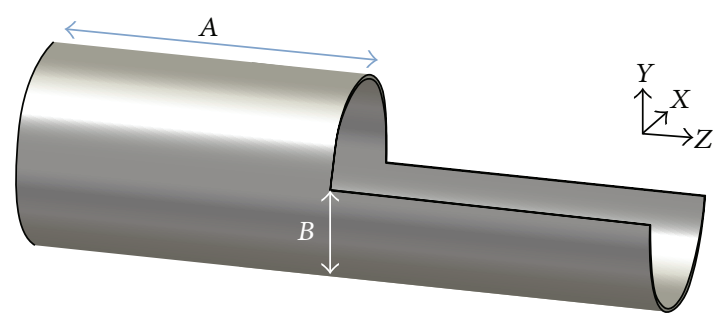

(b) Step-cut design

Figure 1: Configurations and parameters of bevel- and step-cut Vlasov antennas.

the launcher aperture [16]. Moreover, Sealy et al. investigated the use of corrugations with the flare to enhance the pattern performance [17]. Other studies used Vlasov antennas as a component in several systems [18-20]. However, a Vlasov antenna with either the bevel- or step-cut has its own maximum radiation shifted by some angle with respect to the axis of the waveguide. The work by the authors in [21] focused on optimizing the reflector position to bring back the maximum radiation along the axis of the waveguide antenna.

In this paper, we first design a bevel-cut Vlasov antenna operating at $3 \mathrm{GHz}$ with the aim of obtaining maximum gain. Next, a step-cut version is designed to have radiation in the same direction as the bevel-cut counterpart, and a comparison of the performance of both is conducted. Later, a new cut shape, better suitable for the use of Vlasov antennas in HPM applications, is presented and its advantages are reported. Furthermore, the optimized reflector position for Vlasov antennas is presented, which will help, with the proper rotation angle, to orient the generated waves along the $+Z$ direction, which is the axis of the waveguide in our case. In addition, with our proposed configuration, the reflector is directly attached to the waveguide structure, decreasing the size of the usual Vlasov antennas with reflectors, and eliminating the need of extra components to hold the waveguide and reflector together. The proposed reflector is applied to bevel-cut and step-cut Vlasov antennas and then further enhanced with curved edges to be applied to the curved cut to evaluate its performance.

\section{Vlasov Antennas}

Both step- and bevel-cut Vlasov antennas are the result of shaping the end part of a circular waveguide. For operation at $3 \mathrm{GHz}$, the used circular waveguide has a radius of $45 \mathrm{~mm}$ and a length of $300 \mathrm{~mm}$.

2.1. Vlasov with Bevel Cut. A Vlasov antenna with a beveled cut is shown in Figure 1(a). The cut angle $\alpha$ is the single parameter available for optimization, and it has the main effect on the gain and radiation patterns of the antenna. The angle that maximizes the gain of the antenna is given by [4]

$$
\alpha=\sin ^{-1}\left(\frac{\left(\rho_{0 n} \lambda\right)}{(2 \pi a)}\right)
$$

where $\rho_{0 n}$ is the $n$th root of the equation $J_{0}\left(\rho_{0 n}\right)=0, \lambda$ is the wavelength, $a$ is the inner radius of the waveguide, and $J_{0}$ is the Bessel function of the first kind and zeroth order.

For the $\mathrm{TM}_{01}$ circular waveguide designed for $3 \mathrm{GHz}, a=$ $4.5 \mathrm{~cm}$ and $\lambda=10 \mathrm{~cm}$. Also, $\rho_{01}=2.405$, so the bevel cut will be calculated as follows:

$$
\alpha=\sin ^{-1}\left(\frac{(2.405 \times 10)}{(2 \pi \times 4.5)}\right)=58.32^{\circ} .
$$

The highest gain according to the equation is obtained at a cut angle of $58.32^{\circ}$. This result has been verified by simulations using ANSYS HFSS. For this angle, the resulting peak gain is $10.9 \mathrm{~dB}$. The gain patterns, computed in CST MWS for both $\phi=0^{\circ}$ and $\phi=90^{\circ}$ planes, are shown in Figure 2. Maximum radiation is obtained in the $\theta=32^{\circ}$ and $\phi=90^{\circ}$ direction.

2.2. Vlasov with Step Cut. A Vlasov antenna with a beveled cut is shown in Figure 1(b). The step cut is determined by the two parameters, $A$ and $B$, as indicated. The value of $A$ is fixed at $148.5 \mathrm{~mm}$, which is the same value obtained with the bevel cut after finding the angle $\alpha$. For comparison purposes, the step-cut Vlasov is designed so that it has the same direction of maximum radiation obtained with the beveled cut. For this purpose, via CST simulations, $B$ is found to be $25 \mathrm{~mm}$. It was noticed that, as the value of $B$ decreases, the angle of maximum radiation increases. The gain patterns of the stepcut Vlasov antenna, in the $\phi=0^{\circ}$ and $\phi=90^{\circ}$ planes, are shown in Figures 3(a) and 3(b), respectively. As is the design aim, the main lobe peaks at $\theta=32^{\circ}$.

Comparing the two Vlasov antenna types, it is verified that the step cut gives better performance in terms of maximum gain and half-power beamwidth (HPBW). However, the beveled cut results in lower sidelobes, and this comes in addition to its suitability for HPM applications.

\section{Improved Cut}

The conventional Vlasov antennas may suffer from electric breakdown when they radiate at HPM. Although the beveled cut was introduced to avoid the sharp edges present in the step cut, it results in a decreased gain and an increased HPBW of the antenna. A new cut shape, introduced by the authors in [22], is shown in Figure 4. This shape, totally based on curves, 


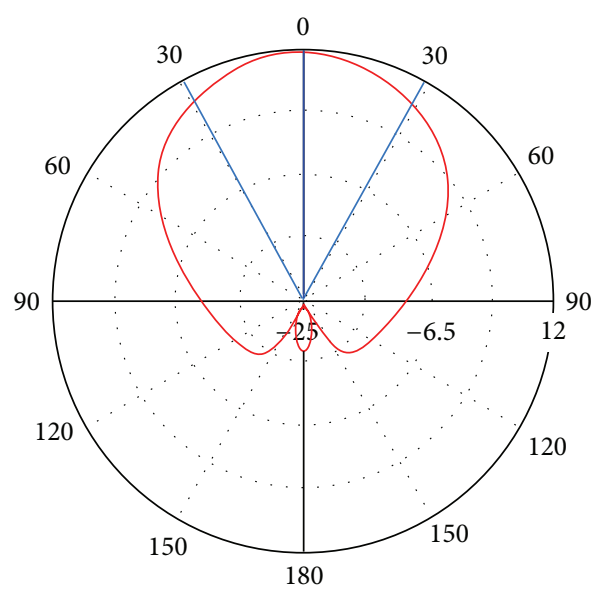

(a) $\phi=0^{\circ}$ plane

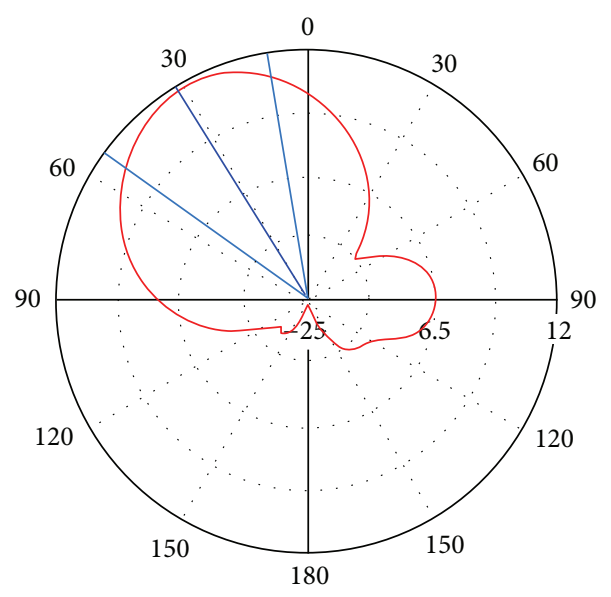

(b) $\phi=90^{\circ}$ plane

FIgURE 2: Gain patterns of bevel-cut design (using CST).

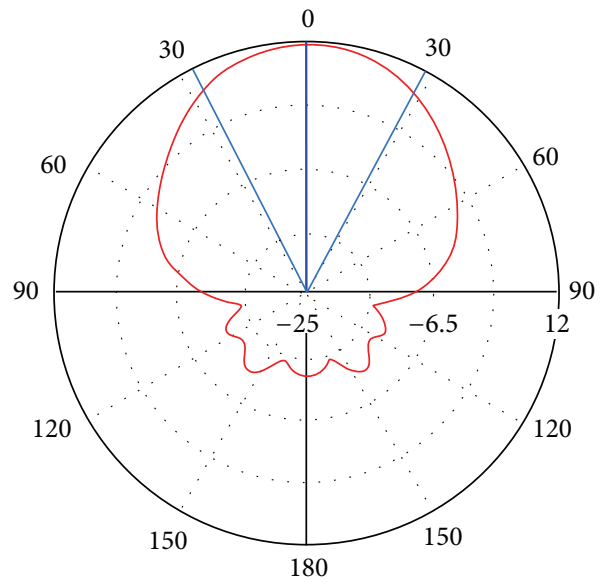

(a) $\phi=0^{\circ}$ plane

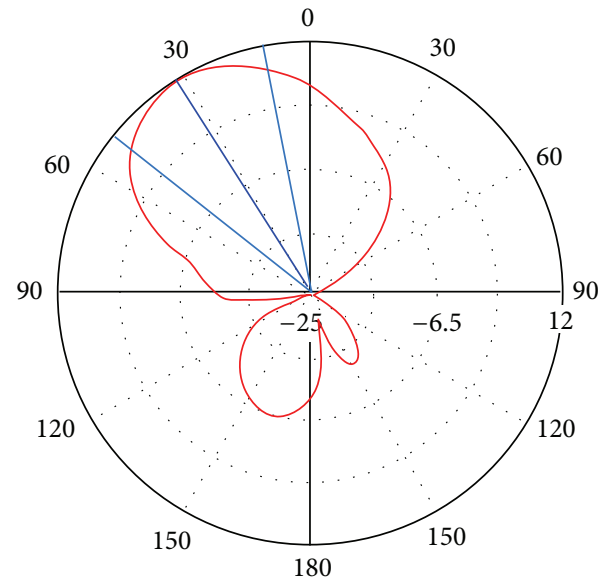

(b) $\phi=90^{\circ}$ plane

Figure 3: Gain patterns of step-cut design (using CST).

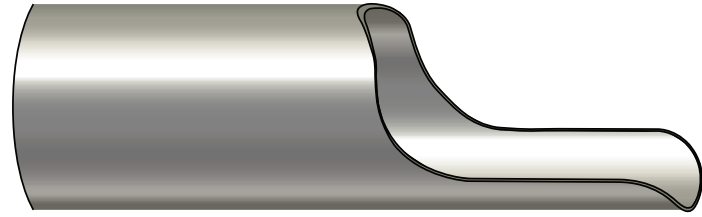

(a) $3 \mathrm{D}$ view

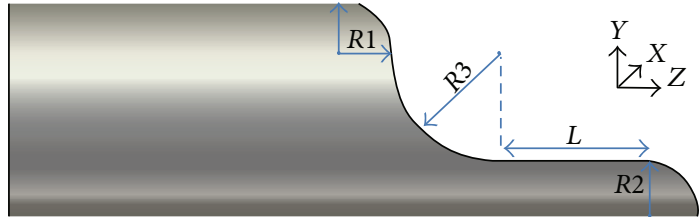

(b) Parametrized dimensions

FIGURE 4: Vlasov antenna with proposed cut. goes ahead of the beveled cut in removing the sharp edges and corners and is a result better suitable for applications involving HPM.

In this design, we have the flexibility to optimize several parameters to reach the desired gain, HPBW, and direction of maximum radiation. These are the radius of Curve $1(R 1)$, the radius of Curve $2(R 2)$, the radius of Curve $3(R 3)$, and the separation between Curves 2 and 3 , noted by $L$ on Figure 4 .

To compare it with the step- and bevel-cut versions, a Vlasov antenna based on the same waveguide and on the proposed cut is designed so that it has the maximum radiation in the same $\theta=32^{\circ}$ and $\phi=90^{\circ}$ direction. Table 1 lists the obtained parameters of the new design. The resulting patterns are shown in Figure 5.

The gain patterns resulting from the step, the beveled, and the proposed Vlasov cuts are compared in Figure 6. The 


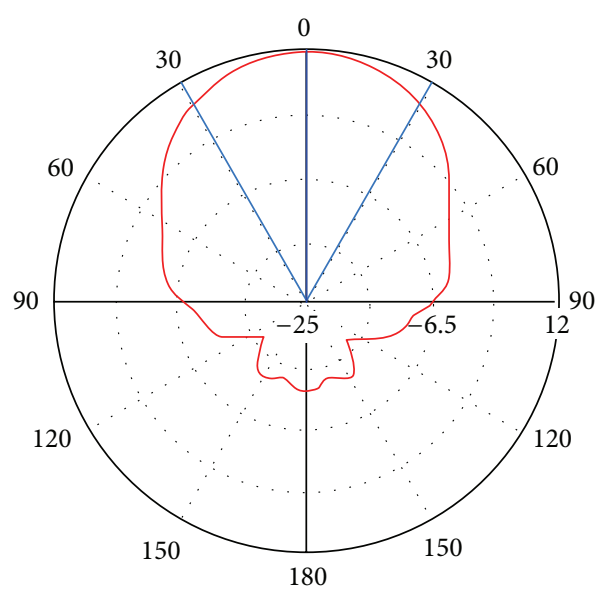

(a) $\phi=0^{\circ}$ plane

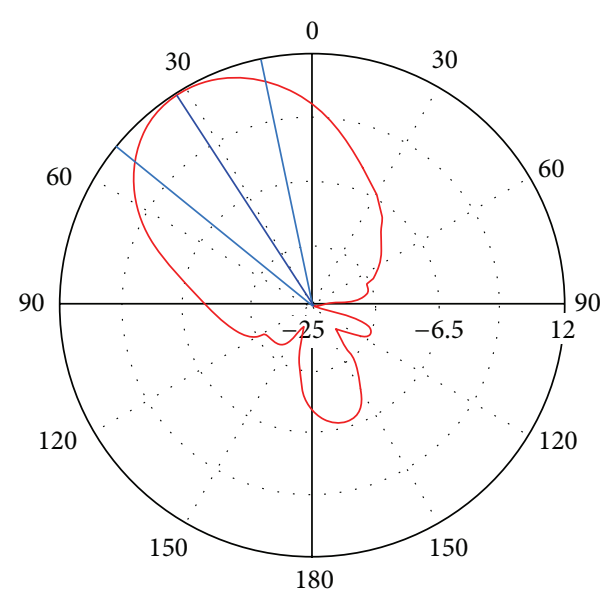

(b) $\phi=90^{\circ}$ plane

FIGURE 5: Gain patterns of the proposed Vlasov antenna (using CST).

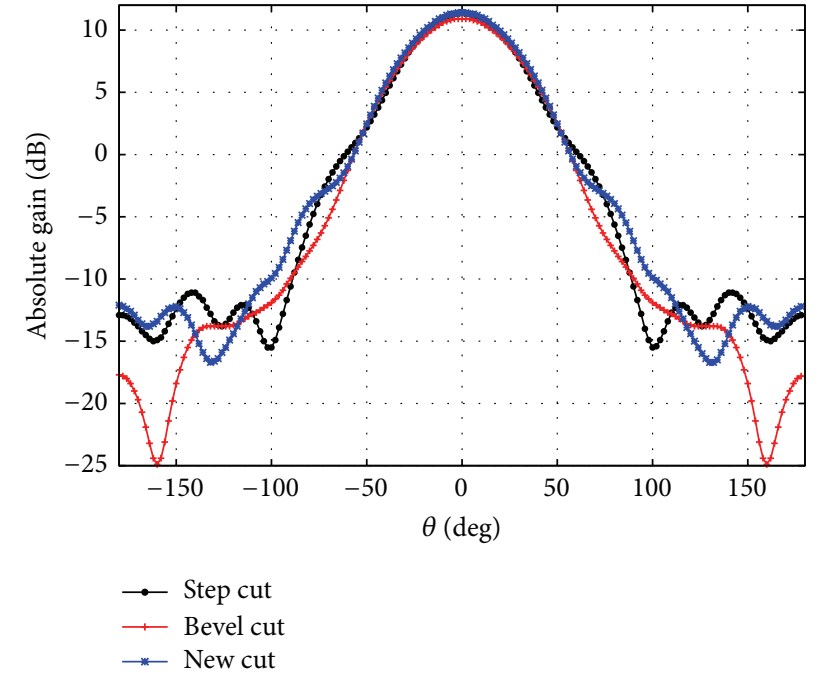

(a) $\phi=0^{\circ}$ plane

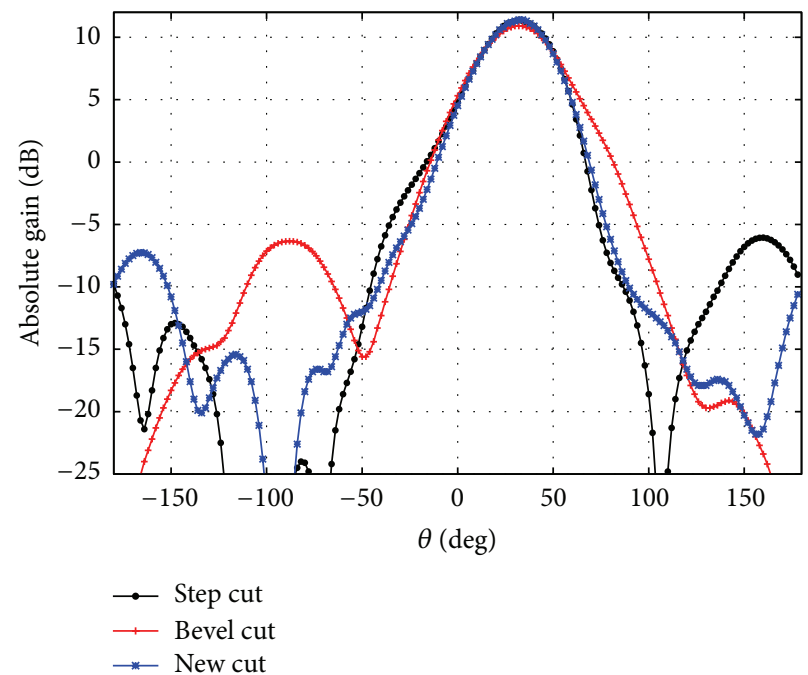

(b) $\phi=90^{\circ}$ plane

FIGURE 6: Comparison of the gain patterns of the step, the beveled, and the proposed Vlasov cuts.

TABLE 1: Parameters of the proposed Vlasov antenna.

\begin{tabular}{lc}
\hline Parameter & Value $(\mathrm{mm})$ \\
\hline Waveguide radius & 45 \\
Waveguide length & 300 \\
$R 1$ & 24.5 \\
$R 2$ & 24.5 \\
$R 3$ & 41 \\
$L$ & 65 \\
\hline
\end{tabular}

obtained peak gain values are given in Table 2. Both CST and HFSS reveal that the proposed Vlasov antenna has a higher peak gain, which overcomes the decreased gain issue that the bevel cut has.

The compared HPBW and sidelobe level ratio (SLR) results, computed in CST, are listed in Table 2. In addition to totally eliminating the sharp edges and corners that limit the performance of the antenna at high powers and providing higher peak gains, the results have shown that the proposed cut gives a smaller HPBW and a better SLR in the $\phi=90^{\circ}$ plane, a better SLR compared to the step cut in the $\phi=0^{\circ}$ plane, and a slightly narrower beam in the $\phi=0^{\circ}$ plane when compared to the bevel cut. These observations are also verified using HFSS simulations.

The reflection coefficient plots, for the three cut types, are given in Figure 7. As shown, the three antennas operate at and around $3 \mathrm{GHz}$ with very low $S 11$ values.

\section{Vlasov with Reflector}

Vlasov antennas radiate waves with maximum radiation obtained in a shifted direction. This was proven through our simulations in Section 2, for which the three designed 
TABLE 2: Compared peak gain, HPBW, and sidelobe level ratio (SLR).

\begin{tabular}{lcccccc}
\hline \multirow{2}{*}{ Antenna } & \multicolumn{2}{c}{ Gain $(\mathrm{dB})$} & \multicolumn{2}{c}{$\mathrm{HPBW}^{\circ}$} & \multicolumn{2}{c}{ SLR (dB) } \\
& CST & HFSS & $\phi=0^{\circ}$ plane & $\phi=90^{\circ}$ plane & $\phi=0^{\circ}$ plane & 24.7 \\
Bevel cut & 10.88 & 10.30 & 58.4 & 44.5 & 40.1 & 22.4 \\
Step cut & 11.33 & 10.64 & 55 & 39 & 23.5 & 17.2 \\
Curved cut & 11.40 & 10.74 & 58 & 39.5 & 18.6 \\
\hline
\end{tabular}

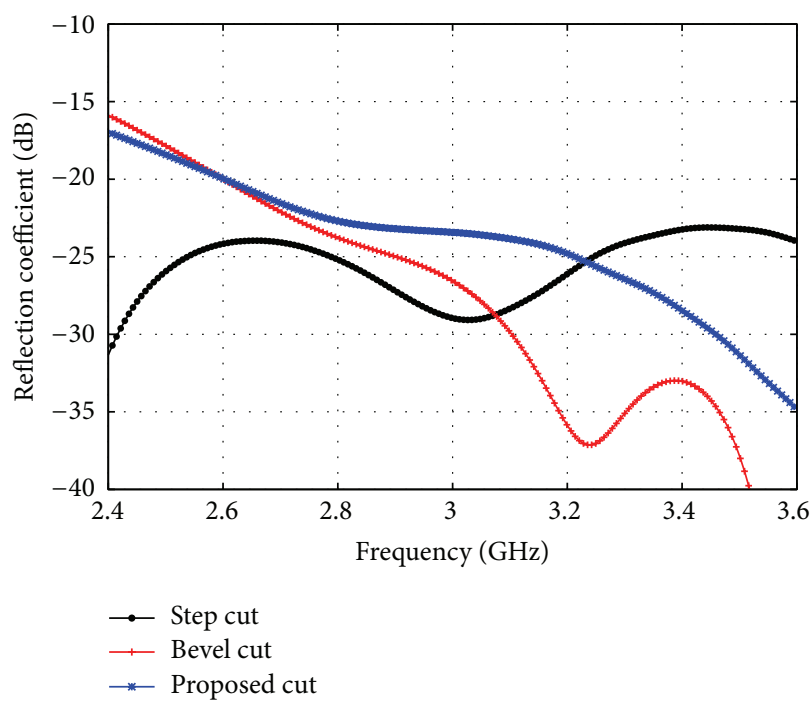

(a) S11 using CST

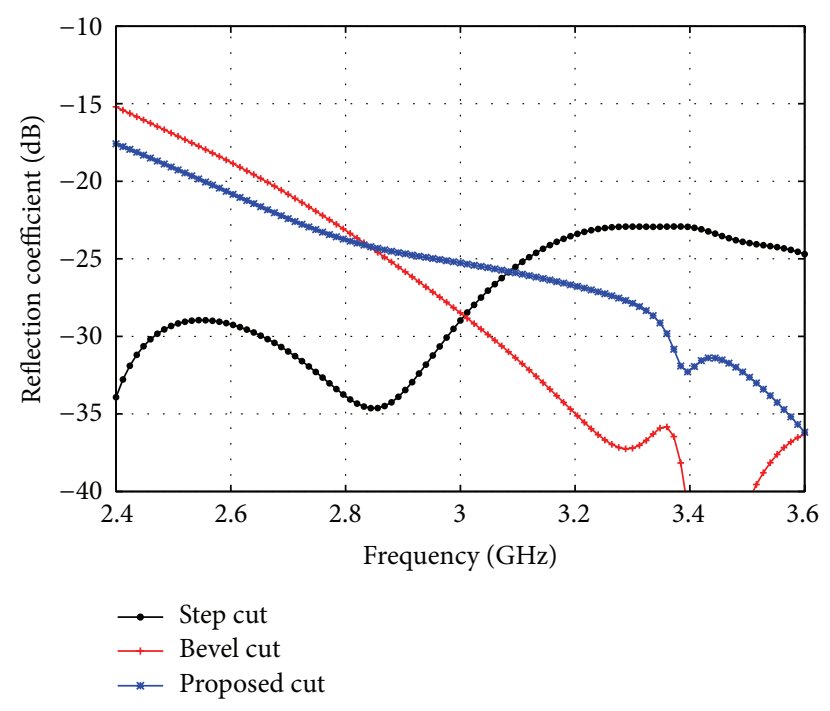

(b) S11 using HFSS

FIGURE 7: Comparison of the reflection coefficient, using both CST and HFSS.

antennas radiate in the shifted directions corresponding to $\theta=\theta_{m}=28^{\circ}$ and $\phi=90^{\circ}$, computed using HFSS. However, it is preferred to have the maximum radiation of the antenna directed in a well-known and easy-to-locate direction. This refers to the axis of the waveguide in our case. In addition, the HPBW in $\phi=0^{\circ}$ plane is large for the three radiated antennas, and it is hard to highly increase the gain of these antennas. In order to achieve rotation of the maximum point of radiation of any of the Vlasov antennas and the curvedcut shape antenna and decrease the HPBW in $\phi=0^{\circ}$ plane, an optimized reflector is proposed in the following work. The reflector has the shape of a half-hollow cylinder. A conventional reflector is used with the step- and bevelcut antennas designed, whereas for the optimized curvedcut shape proposed in Section 3 an optimized reflector having also curved edges instead of the sharp corners of the conventional reflector is proposed. In both cases, the two reflectors have several parameters that affect the radiation: the starting point of the reflector, the radius of the reflector, the length of the reflector, and the angle of rotation of the reflector with respect to the waveguide axis. All these parameters have been optimized to obtain the desired radiation patterns.

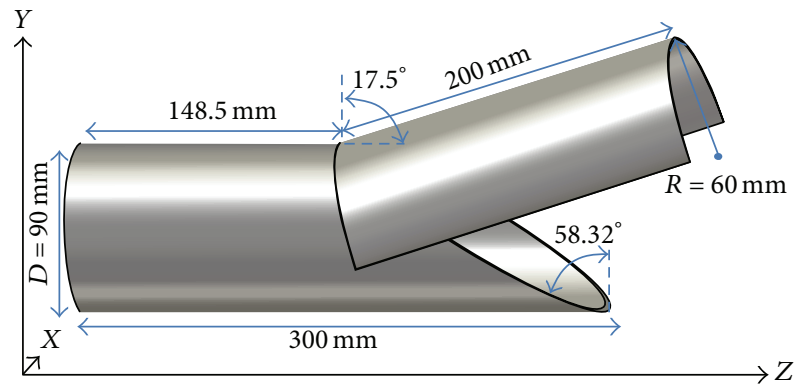

FIgURE 8: Bevel-cut Vlasov antenna with reflector.

\subsection{Bevel-Cut Vlasov Antenna with Reflector}

4.1.1. Design and Simulations. Using the bevel-cut antenna design in Section 2.1, the maximum radiation is obtained in the shifted direction corresponding to $\theta=\theta_{m}=28^{\circ}$ and $\phi=90^{\circ}$, computed using HFSS. A conventional reflector having the shape of a half-hollow cylinder is then attached to the bevel-cut Vlasov antenna as shown in Figure 8. The added reflector has the optimized values of $60 \mathrm{~mm}$ for the cylinder 


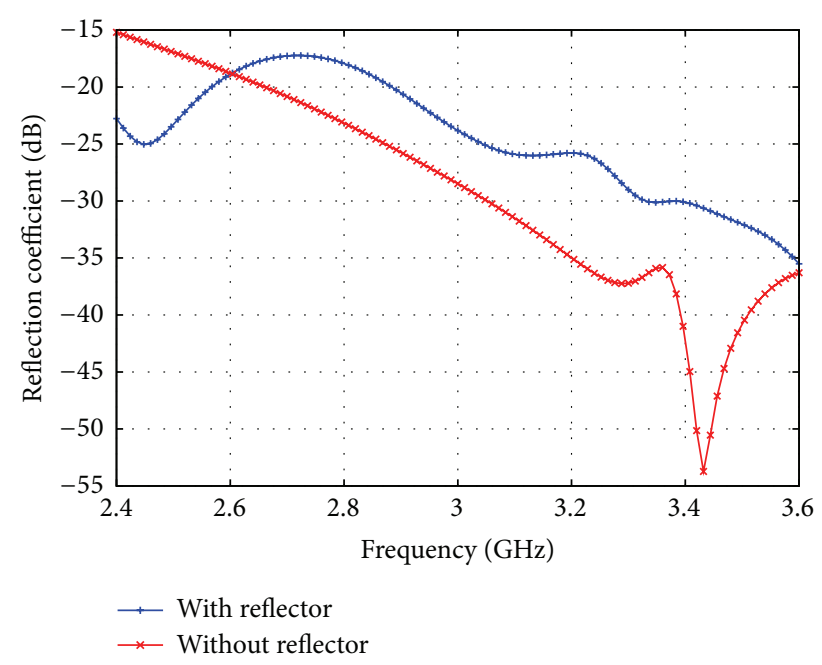

FIGURE 9: Reflection coefficient computed using HFSS, with noreflector case shown in red (dashed), and with reflector in blue.

radius and a length of $200 \mathrm{~mm}$. Upon rotating the reflector by a specific angle, it is seen that as the angle increases the shift angle approaches origin. The initial bevel-cut Vlasov antenna gives a maximum computed gain of $10.3 \mathrm{~dB}$, with the maximum radiation along the $\theta=28^{\circ}$ and $\phi=90^{\circ}$ direction. The optimized reflector angle for perfect direction along the $+Z$ axis is seen at angle of $17.5^{\circ}$. For this angle, the maximum radiation is back along the axis of the waveguide; that is, $\theta=0^{\circ}$ and $\phi=90^{\circ}$. The bevel-cut antenna operates at $3 \mathrm{GHz}$ as shown in the reflection coefficient plot $\left(S_{11}\right)$ in Figure 9. It has a gain of $10.9 \mathrm{~dB}$ and a reduced HPBW, as indicated in Figures 10(a) and 10(b). It is shown that, for the case with the reflector, the maximum radiation is redirected along the axis of the waveguide.

4.1.2. Verification of the Results Using CST. The same design simulated in Section 4.1 using HFSS has been also tested using CST. The gain patterns in the two cases (without and with reflector) are shown in Figure 11. As can be seen, the maximum gain of the antenna is redirected along the axis of the waveguide. The $3 \mathrm{D}$ gain patterns comparing the two cases are shown in Figure 12. Furthermore, the peak gain has increased from 10.88 to $12 \mathrm{~dB}$, and the HPBW has decreased in the plane of interest, that is, $\phi=0^{\circ}$ plane, from $58.4^{\circ}$ to $41.7^{\circ}$.

\subsection{Step-Cut Vlasov Antenna}

4.2.1. Design and Simulations. Again, using the step-cut antenna design in Section 2.2, the maximum radiation is obtained in the shifted direction corresponding to $\theta=\theta_{m}=$ $28^{\circ}$ and $\phi=90^{\circ}$, computed using HFSS. The same reflector used in Section 4.1 is then attached to the step-cut antenna as shown in Figure 13. Here, $L$ is the distance between the waveguide port and the start of the reflector. The gain patterns of the designed step-cut antenna are shown in Figure 14. By inspecting Figure 14(b), the concept of rotating the reflector is validated and maximum radiation is obtained back along the waveguide axis for a rotation angle of $17.5^{\circ}$ similar to the one used for the bevel-cut case. The design has been also simulated using CST showing the same results with an increment in gain from $11.33 \mathrm{~dB}$ to $11.86 \mathrm{~dB}$ with a large decrement in HPBW in the plane of interest $\left(\phi=0^{\circ}\right)$ from $55^{\circ}$ to $41^{\circ}$ with negligible changes in the $\phi=90^{\circ}$ plane.

\section{Improved Curved Cut with Optimized Reflector Position}

After modifying the cut of the Vlasov antenna in order to optimize its performance as described in Section 3, in this section the optimized reflector proposed in Section 4 will be used to validate the concept of rotating the reflector in order to radiate the wave generated along the $+Z$ direction on the novel cut shape. However, in order to also avoid having sharp corners in the reflector, an optimized one is proposed here to be attached to the curved-cut shape antenna. Figure 15 shows the proposed combined antenna joining the curvedcut antenna and the curved-edges reflector with its optimized parameters. The parameters of the curved-cut antenna are the same as those used in the previous design in Section 3 and are listed in Table 1. In order to get the optimized values of the reflector radius and length indicated, the following parametric study has been done.

\subsection{Parametric Study}

5.1.1. Radius Study. In order to choose the appropriate value of the reflector radius, the radius has been varied from $40 \mathrm{~mm}$ to $70 \mathrm{~mm}$. The realized gain value at each value of radius is shown in Figure 16(a), and the gain pattern in the plane of interest $\left(\phi=90^{\circ}\right)$ at each radius value is also shown in Figure 16(b).

Inspecting Figure 16(a), it is seen that the realized gain in the plane of interest $\left(\phi=90^{\circ}\right)$ increases as the radius of the reflector increases, till reaching a maximum value at a radius of $65 \mathrm{~mm}$. However, inspecting the gain pattern in the $\phi=$ $90^{\circ}$ plane in Figure 16(b), it is seen that at this radius the SLL and HPBW are higher than those with the second maximum value of gain at a radius of $60 \mathrm{~mm}$. Hence a radius of $60 \mathrm{~mm}$ is suggested to be the optimized value taking into account the value of gain, SLL and HPBW.

5.1.2. Length Study. In order to choose the appropriate value of the reflector length, the radius has been varied from $150 \mathrm{~mm}$ to $220 \mathrm{~mm}$. The realized gain value at each value of length is shown in Figure 17(a), and the gain pattern in the plane of interest $\left(\phi=90^{\circ}\right)$ at each length value is also shown in Figure 17(b).

Inspecting Figure 17(a), it is seen that the realized gain in the plane of interest $\left(\phi=90^{\circ}\right)$ increases as the length of the reflector increases, till reaching an approximate maximum value at a length of $200 \mathrm{~mm}$, after which the value of gain might increase but in very small steps compared to the increase in the length. In addition, inspecting the gain pattern in the $\phi=90^{\circ}$ plane in Figure 17(b), it is seen that at this chosen value of length the SLL and HPBW are better than 


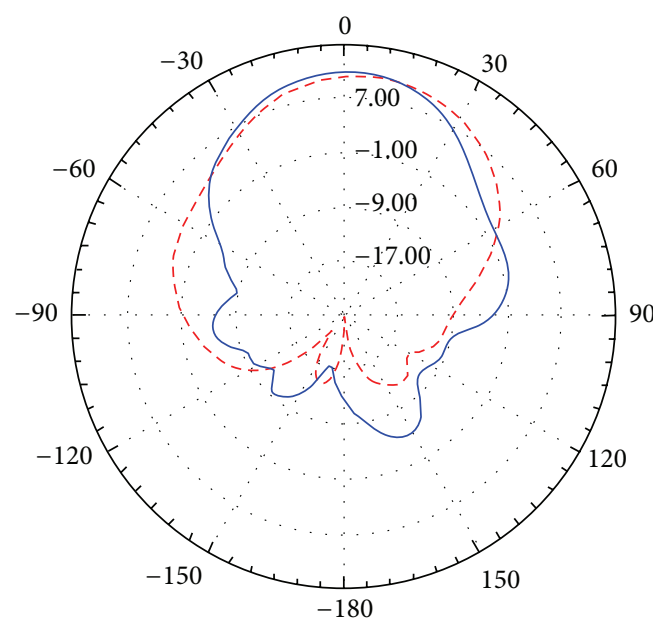

(a) Red in the plane formed by the $X$-axis and the point of maximum radiation $\left(\theta=\theta_{m}, \phi=90^{\circ}\right)$, blue in $\phi=0^{\circ}$ plane

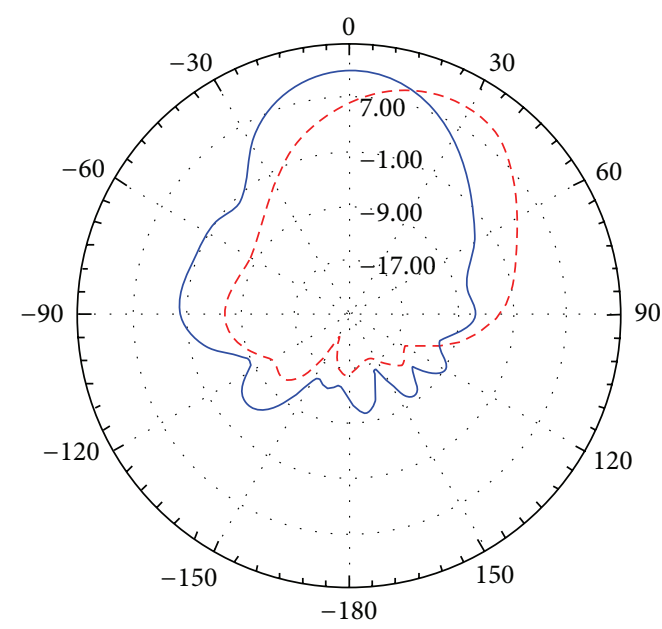

(b) $\phi=90^{\circ}$ Plane

FIGURE 10: Simulated gain patterns computed using HFSS, with initial bevel-cut results shown in red (dashed) and proposed design results in blue.

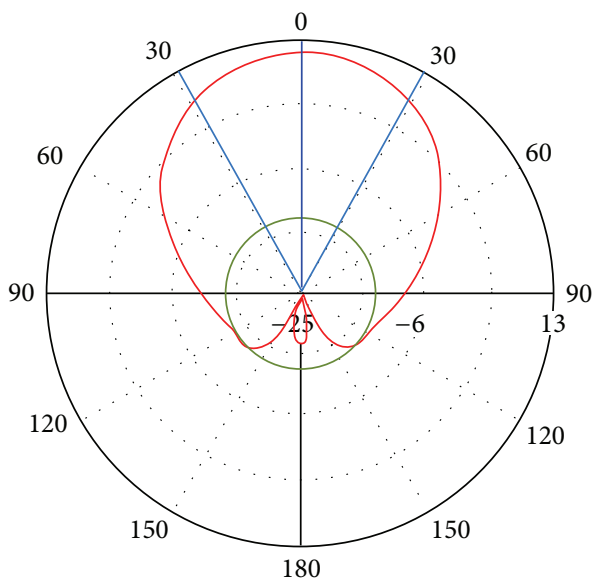

(a) $\theta=\theta_{m}, \phi=90^{\circ}$ plane

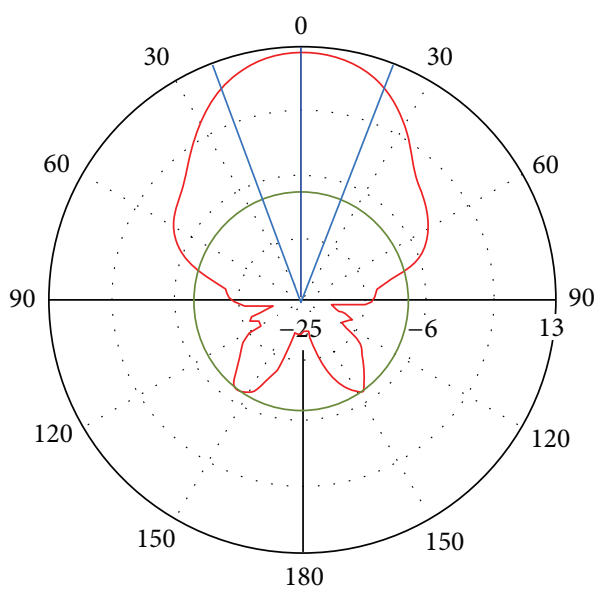

(c) $\phi=0^{\circ}$ plane

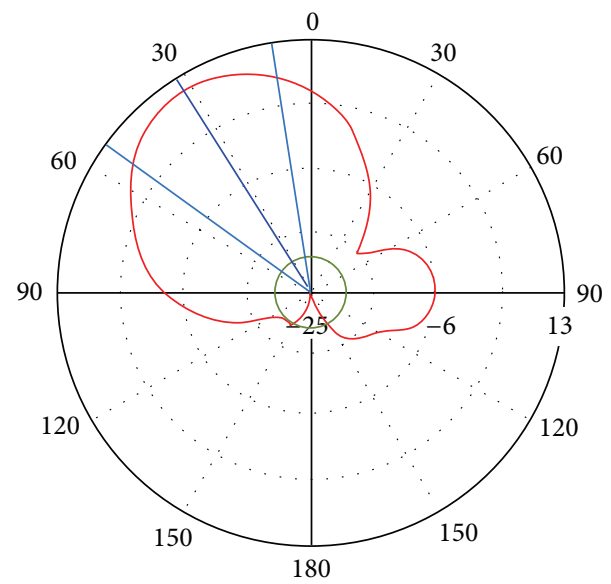

(b) $\phi=90^{\circ}$ plane

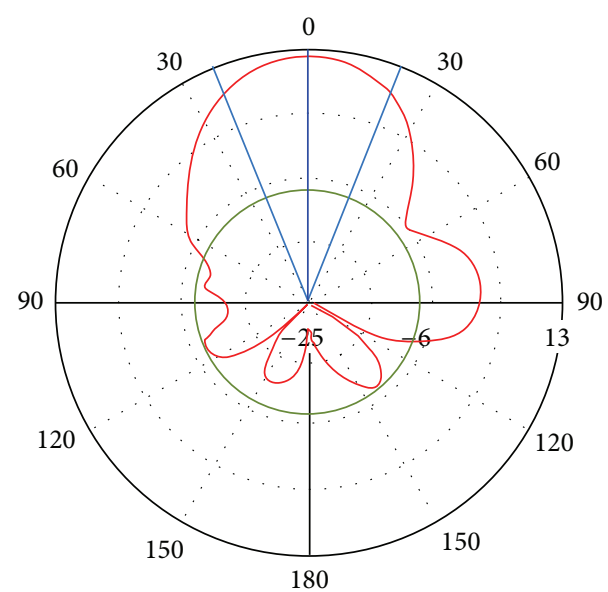

(d) $\phi=90^{\circ}$ plane

FIGURE 11: Bevel-cut simulated gain patterns using CST: (a) and (b) without adding the reflector and (c) and (d) after adding the reflector. 


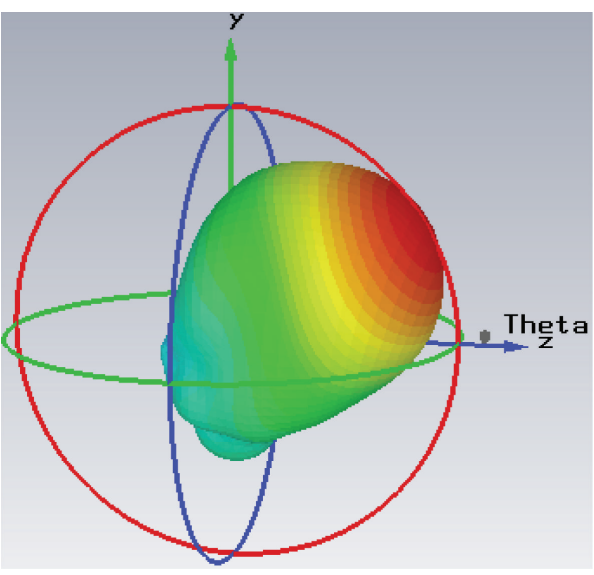

(a) Without reflector

FIGURE 12: Bevel-cut 3D gain patterns using CST: (a) without reflector and (b) with reflector.

(b) With reflector

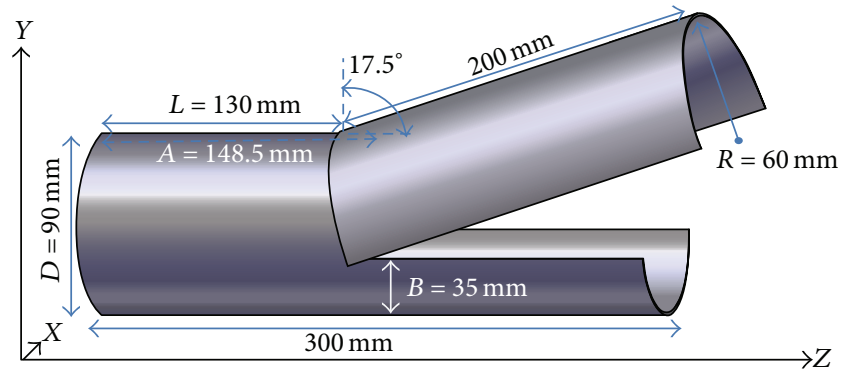

FIGURE 13: Step-cut Vlasov antenna with a reflector.

those with the higher values. Hence a length of $200 \mathrm{~mm}$ is suggested to be the optimized value taking into account the value of gain, SLL, HPBW, and antenna structure size.

5.2. Simulations and Results. Taking into account the optimized values of the reflector parameters, the proposed combined antenna suitable for high power microwave applications has been simulated using CST with the radiation pattern results shown in Figure 18.

The maximum radiation of the curved-cut antenna without using the reflector was shifted in the direction corresponding to $\theta=\theta_{m}=28^{\circ}$ and $\phi=90^{\circ}$, as depicted in Section 3. Inspecting Figure 18, the concept of rotating the reflector is validated and the radiation in $\phi=90^{\circ}$ plane is now directed towards the $+Z$ plane, as can be seen in Figure 18(b). In addition, the proposed combined antenna also increases the gain of the antenna from $11.4 \mathrm{~dB}$ to $11.92 \mathrm{~dB}$ and decreases its HPBW in the $\phi=0^{\circ}$ plane from $58^{\circ}$ to $41^{\circ}$, with minor changes in the $\phi=90^{\circ}$ plane. The same result has been also achieved using HFSS with an increment in gain from $10.7 \mathrm{~dB}$ to $11.00 \mathrm{~dB}$ as shown in Figure 19.
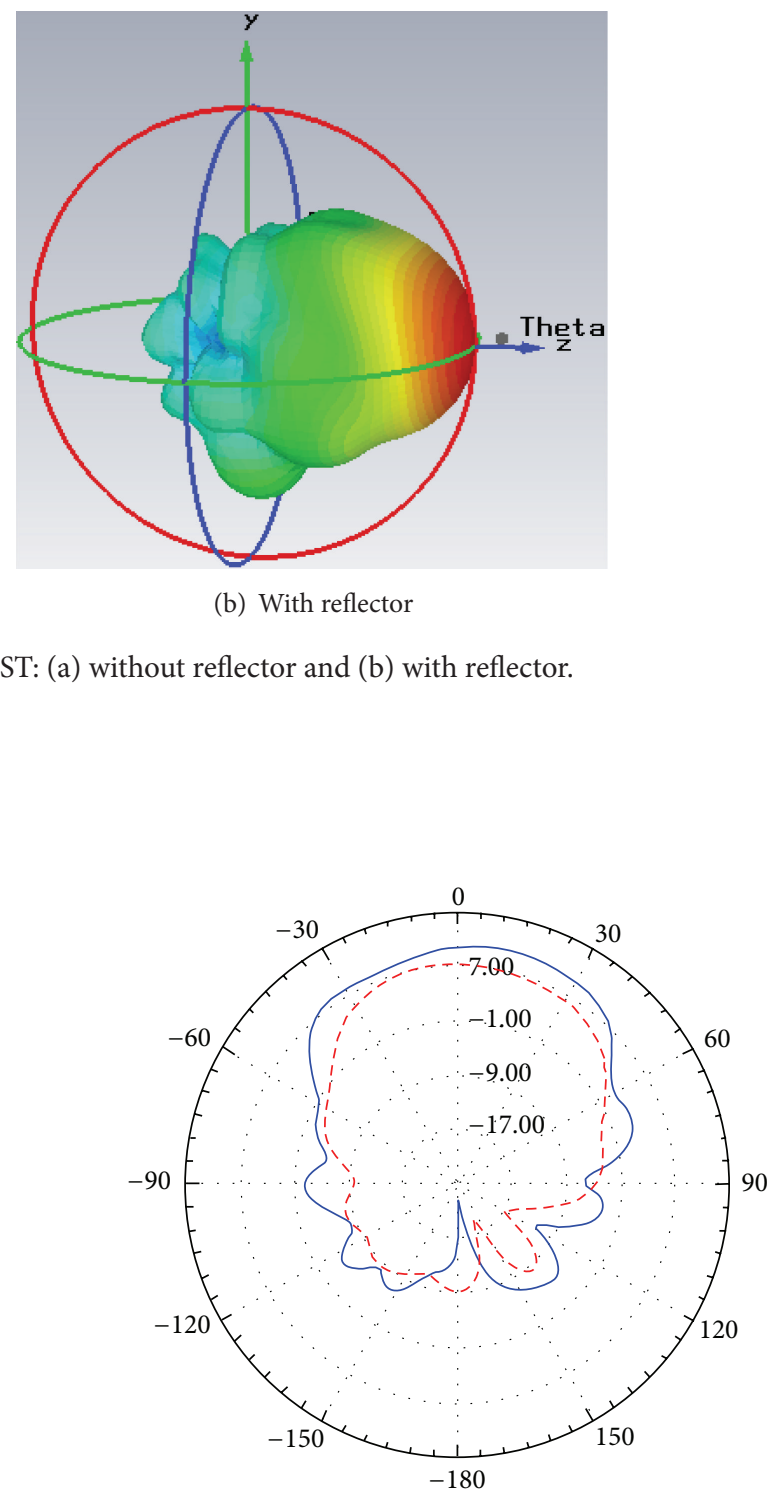

(a) Red in $\theta=\theta_{m}, \phi=90^{\circ}$ plane, blue in $\phi=0^{\circ}$ plane

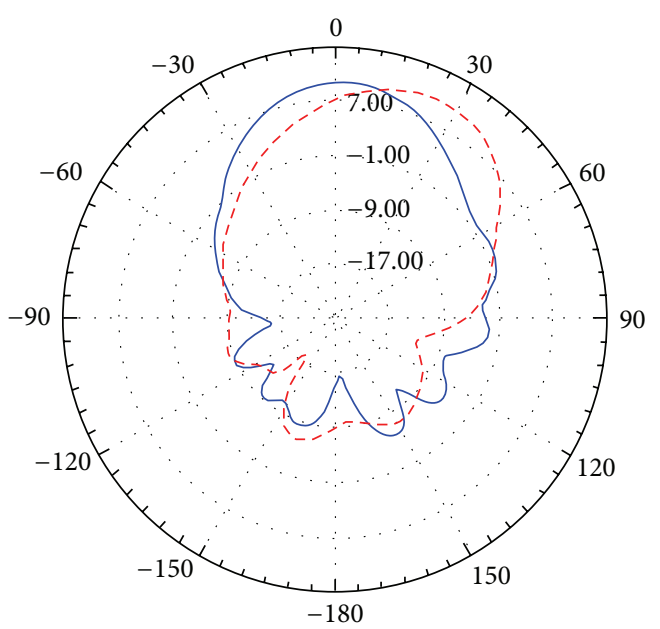

(b) $\phi=90^{\circ}$ plane

FIGURE 14: Step-cut simulated gain patterns, with initial step-cut results shown in red (dashed) and proposed design results in blue. 


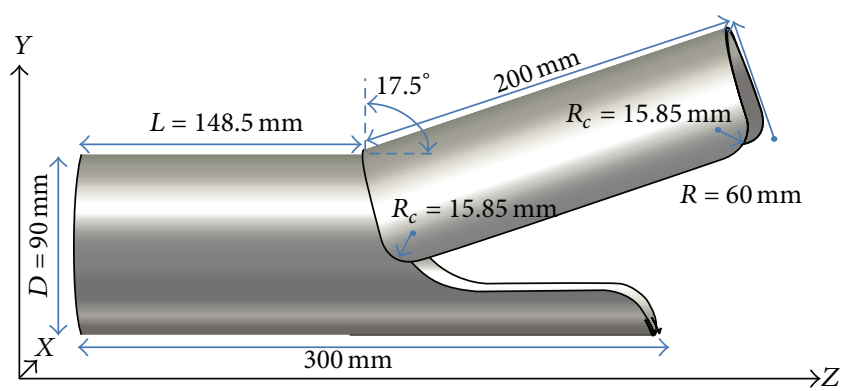

FIGURE 15: Enhanced Vlasov cut with a rotated optimized reflector with curved edges.

TABLE 3: Comparison of the radiation characteristics of the three designed antennas, with and without having the enhanced suitable reflector computed using CST.

\begin{tabular}{lcccc}
\hline \multirow{2}{*}{ Antenna } & \multirow{2}{*}{ Reflector } & \multicolumn{2}{c}{$\mathrm{HPBW}^{\circ}$} & Gain $(\mathrm{dB})$ \\
& & $\phi=0^{\circ}$ plane & $\phi=\phi_{m}^{\circ}$ plane & \\
\hline \multirow{2}{*}{ Bevel cut } & Without & 58.4 & 44.5 & 10.88 \\
& With & 41.7 & 42.9 & 12 \\
\hline \multirow{2}{*}{ Step cut } & Without & 55 & 40.1 & 11.33 \\
& With & 42 & 43 & 11.86 \\
\hline \multirow{2}{*}{ Curved cut } & Without & 58 & 39 & 11.40 \\
& With & 41.1 & 41 & 11.92 \\
\hline
\end{tabular}

Combining all the simulations of the three designed antennas, with and without having the enhanced suitable reflector, Table 3 compares the radiation characteristics of the various cases studied. As listed, in addition to bringing back the maximum radiation along the axis of the waveguide, the proposed combined antenna also increases the gain of the antenna and decreases its HPBW.

Observations. Several simulations have been done to study the effect of each parameter of the Vlasov antenna with curved-cut and improved reflector position and shape on the radiation performance. The following observations have been inspected.

(i) As the starting point of the reflector goes backward to the start of the Vlasov antenna, the gain decreases and the HPBW highly increases. This change also affects the point of maximum radiation which will be rotated by a certain degree from the axis of the waveguide.

(ii) As the rotation angle of the reflector increases, the gain slowly increases with minor variations in HPBW. However, this change also affects the point of maximum radiation which will be rotated by a certain degree from the axis of the waveguide.

(iii) As the radius of the reflector decreases, the gain slowly decreases and the HPBW increases.

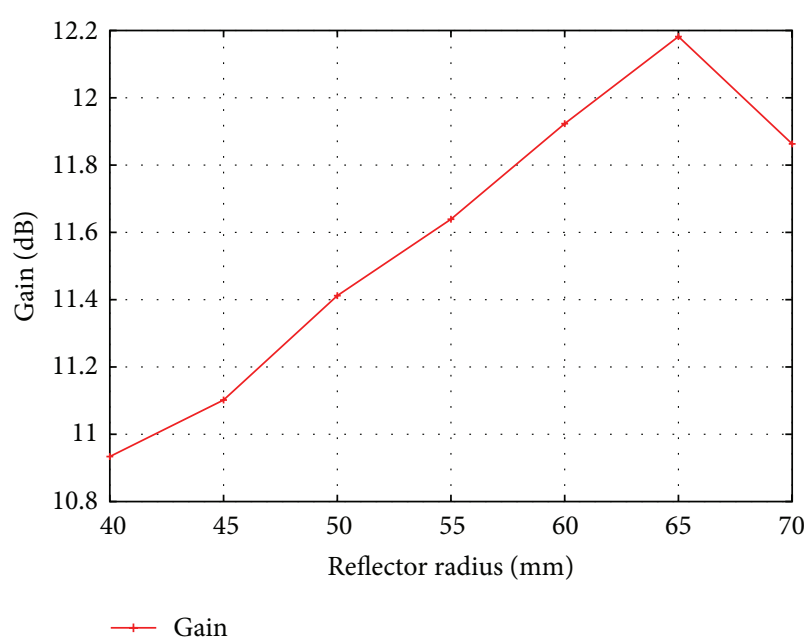

(a)

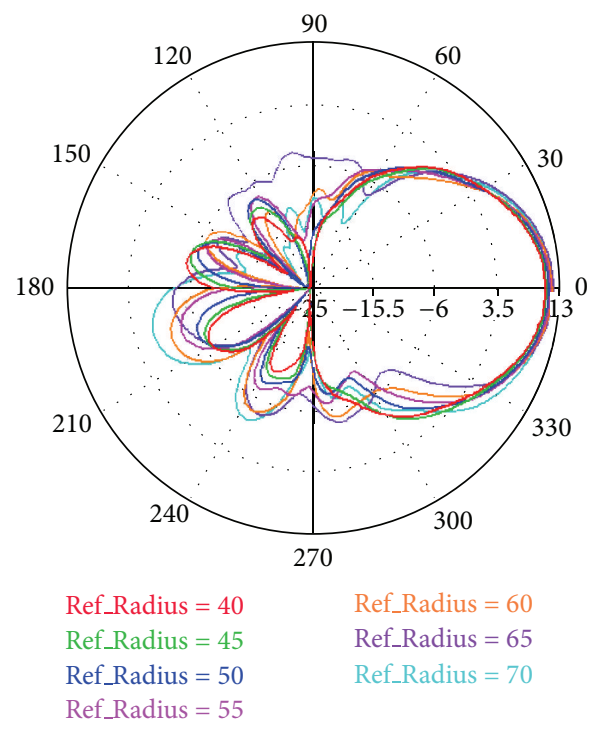

(b)

FIGURE 16: Simulated gain patterns computed using CST: (a) realized gain values and (b) 2D gain patterns.

Hence, care should be taken in choosing the optimized values for each of these parameters in order to attain the desired performance.

One example on the use of such optimizations is to extend the reflector length to $250 \mathrm{~mm}$, decrease the angle of rotation to $15^{\circ}$, and increase the starting point of the reflector (noted by $L$ ) to $151 \mathrm{~mm}$. Applying these changes, the gain will increase to $12.42 \mathrm{~dB}$ and the HPBW in the $\phi=90^{\circ}$ plane will decrease to $37^{\circ}$, with the HPBW in the $\phi=0^{\circ}$ kept constant at $41.1^{\circ}$.

\section{Conclusion}

The Vlasov antenna, originally designed with a step cut made to one end of a circular waveguide, has been improved by 


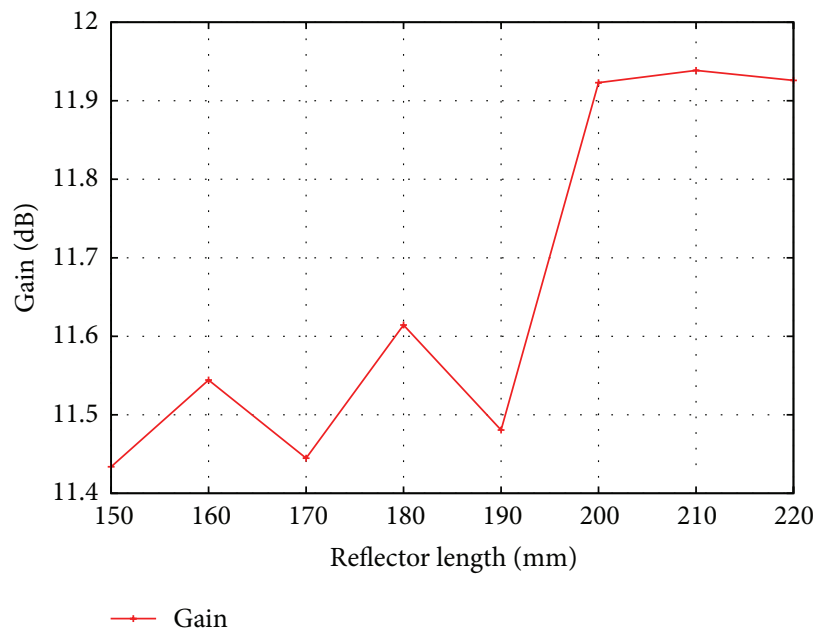

(a)

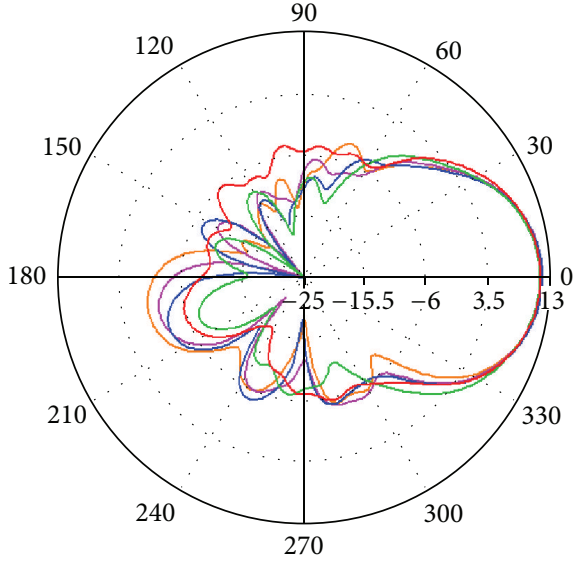

$\begin{array}{ll}\text { Ref_Length }=180 & \text { Ref_Length }=210 \\ \text { Ref_Length }=190 & \text { Ref_Length }=220\end{array}$

Ref_Length $=200$

FIGURE 17: Simulated gain patterns computed using CST: (a) realized gain values, (b) 2D gain patterns.

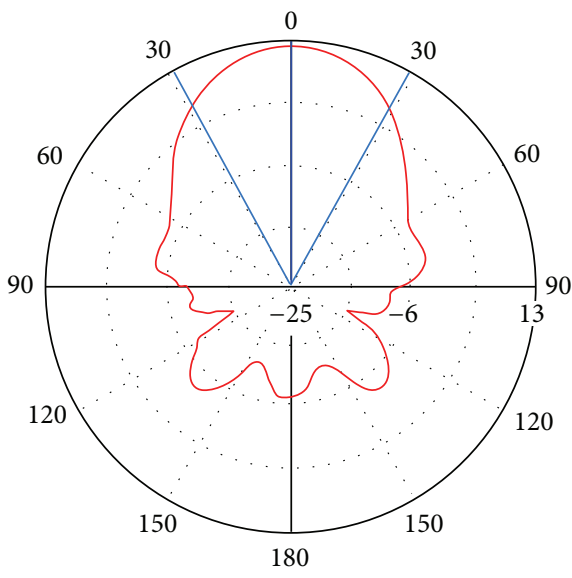

(a) $\phi=0^{\circ}$ plane

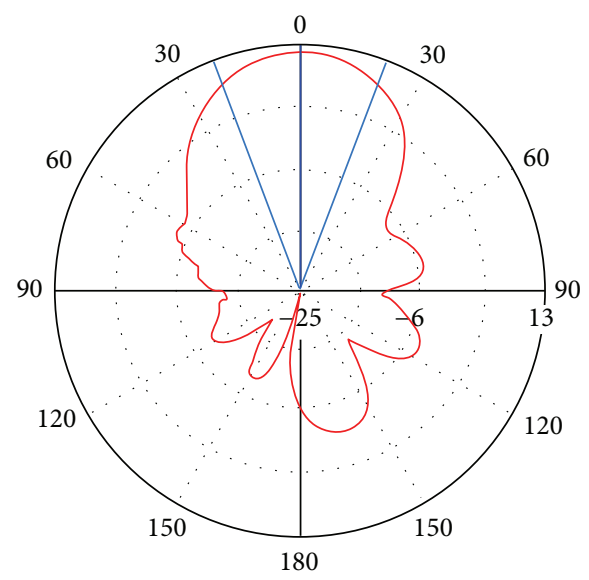

(b) $\phi=90^{\circ}$ plane

FIGURE 18: Gain patterns of the proposed curved-cut antenna with the optimized reflector position and shape, computed using CST.

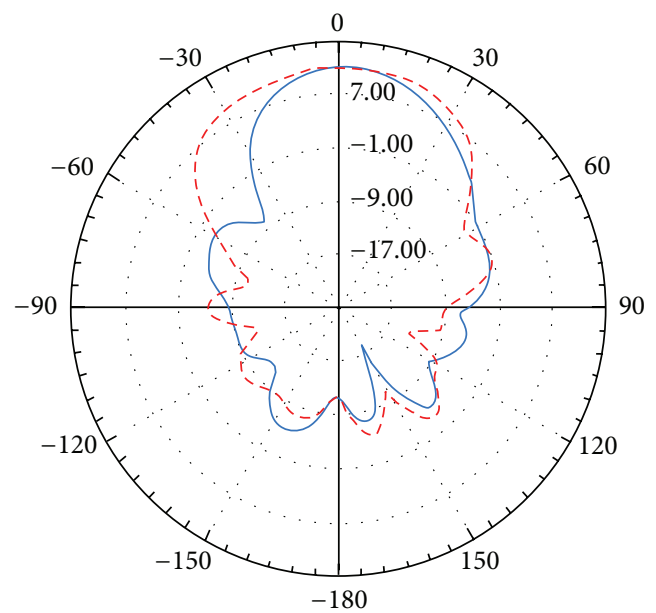

FIGURE 19: Gain patterns of the proposed curved-cut antenna with the optimized reflector position and shape, computed using HFSS: red (dashed) in $\phi=0^{\circ}$ plane and blue in $\phi=90^{\circ}$ plane. 
Nakajima who implemented it using a beveled cut. The latter cut gets rid of the sharp corners present in the former one, which makes the antenna supportive of higher microwave powers, but this comes at the cost of reduced antenna gain and broader beam widths. In addition, the maximum radiation of these two antennas is shifted by some angle in the $\phi=90^{\circ}$ plane, assuming the antenna is directed along the $+Z$ axis. In this paper, an improved shape with curved cuts for high power microwaves applications has been suggested, leading to higher gain, decreased HPBW, and decreased sidelobes levels. Furthermore, two optimized reflectors, one with enhanced position and the second with additional shape enhancement having curved edges, are proposed, with the ability to radiate the maximum radiation back to the $+Z$ axis and further decrease the HPBW. This concept has been validated using the bevel- and step-cut Vlasov antenna, and with the new curved cut proposed in this paper.

\section{Conflict of Interests}

The authors declare that there is no conflict of interests regarding the publication of this paper.

\section{Acknowledgments}

This work is partially supported by an Associated Research Unit (ARU) Fund from the Lebanese National Council for Scientific Research and by the Electromagnetics and Radio Frequency Research Group (EMRF) Fund at the American University of Beirut.

\section{References}

[1] S. N. Vlasov and I. M. Orlova, "Quasioptical transformer which transforms the waves in a waveguide having a circular cross section into a highly directional wave beam," Radiophysics and Quantum Electronics, vol. 17, no. 1, pp. 115-119, 1974.

[2] S. N. Vlasov and I. M. Orlova, "Quasioptical transformer which transforms the waves in a waveguide having a circular cross section into a highly directional wave beam," Radiophysics Quantum Electronics, vol. 17, no. 1, pp. 115-119, 1974.

[3] S. N. Vlasov, L. I. Zagryadskaya, and M. I. Petelin, “Transformation of a whispering gallery mode, propagating in a circular waveguide, into a beam of waves," Radio Engineering and Electronic Physics, vol. 20, no. 10, pp. 14-17, 1975.

[4] G. S. Ling and C. W. Yuan, "Design of a Vlasov antenna with reflector," International Journal of Electronics, vol. 91, no. 4, pp. 253-258, 2004.

[5] H. Zhou and X. Yang, "Design of novel VLASOV-type antennas for high power microwaves (HPM)," in Proceedings of the IEEE 34th International Conference on Plasma Science (ICOPS '07), p. 912, Albuquerque, NM, USA, June 2007.

[6] O. Wada and M. Nakajima, in Proceedings of the EC6 Joint Workshop on ECE and ECRH, Oxford, UK, September 1987.

[7] B. G. Ruth, R. K. Dahlstrom, C. D. Schlesiger, and L. F. Libelo, "Design and low-power testing of a microwave Vlasov mode converter," in Proceedings of the IEEE MTT-S International Microwave Symposium Digest, vol. 3, pp. 1277-1280, June 1989.
[8] C. Vollaire, L. Nicolas, K. A. Connor, S. J. Salon, B. G. Ruth, and L. F. Libelo, "Microwave radiation from slant cut cylindrical antennas-modeling an experiment," IEEE Transactions on Magnetics, vol. 34, no. 5, pp. 2712-2715, 1998.

[9] P. J. Sealy and R. J. Vernon, "Low-power investigation of TE0n and TM0n mode Vlasov launchers (Gyrotron applications)," in Proceedings of the Antennas and Propagation Society International Symposium, vol. 2, pp. 950-953, 1989.

[10] P. J. Sealy and R. J. Vernon, "Equivalence-principle model for radiation from $\mathrm{TE}_{0 n}$ and $\mathrm{TM}_{0 n}$ mode step-cut and slant-cut Vlasov feeds," in Antennas and Propagation Society International Symposium, vol. 3, pp. 1836-1839, London, Canada, 1991.

[11] R. K. Dahlstrom, L. J. Hadwin, B. G. Ruth, and L. F. Libelo, "Reflector design for an X-band Vlasov antenna," in Proceedings of the Antennas and Propagation Symposium Digest, vol. 2, pp. 968-971, May 1990.

[12] M. Fazaelifar and M. R. Fatorehchy, "Design, fabrication and test of parabolic cylinder reector and horn for increasing the gain of vlasov antenna," Progress In Electromagnetics Research Letters, vol. 4, pp. 191-203, 2008.

[13] X. Zhang, Q. Wang, Y. Cheng, and S. Wen, "Design of a 220G Hz Vlasov (antenna) mode converter," in Proceedings of the International Workshop on Microwave and Millimeter Wave Circuits and System Technology (MMWCST '12), pp. 246-247, April 2012.

[14] J. A. Lorbeck and R. J. Vernon, "Singly curved dual-reflector synthesis technique applied to a quasi-optical antenna for a gyrotron with a whispering-gallery mode output," IEEE Transactions on Antennas and Propagation, vol. 39, no. 12, pp. 1733-1741, 1991.

[15] B. Wang, C.-H. Du, P.-K. Liu, Z.-H. Geng, and S.-X. Xu, "P312: study of a Vlasov mode converter for $94 \mathrm{GHz}$ whispering gallery mode gyrotron," in Proceedings of the IEEE International Vacuum Electronics Conference (IVEC '10), pp. 353-354, IEEE, Monterey, Calif, USA, May 2010.

[16] J. Braunstein, K. Connor, S. Salon, L. Libelo, and C. D. Schlesiger, "Analysis of flared end for Vlasov-type antenna: comparison of 2D finite element analysis with experiment," IEEE Transactions on Magnetics, vol. 30, no. 5, pp. 3120-3123, 1994.

[17] P. J. Sealy, R. J. Vernon, and J. A. Lorbeck, "Vlasov feeds with corrugated flares for pattern enhancement," in Proceedings of the IEEE Antennas and Propagation Society International Symposium, vol. 1, pp. 714-717, June 1995.

[18] A. D. R. Phelps, P. R. Winning, and S. N. Spark, "Broadband quasi-optical mode converters for high power microwaves," in IEE Colloquium on Antenna and Propagation Problems of Ultrawideband Radar, pp. 1-5, London, UK, 1993.

[19] T. A. Spencer, C. E. Davis, K. J. Hendricks, R. M. Gilgenbach, and M. J. Arman, "Long-pulse Hyrotron-backward-wave oscillator experiments," in Proceedings of the IEEE International Conference on Plasma Science, 1993.

[20] B. Wang, C.-H. Du, P.-K. Liu, Z.-H. Geng, and S.-X. Xu, "P3-12: study of a Vlasov mode converter for $94 \mathrm{GHz}$ whispering gallery mode gyrotron," in Proceedings of the IEEE International Vacuum Electronics Conference (IVEC '10), pp. 353-354, Monterey, Calif, USA, May 2010.

[21] H. M. El Misilmani, M. Al-Husseini, K. Y. Kabalan, and A. ElHajj, "Optimized reflector position for vlasov antennas," in Proceedings of the Progress in Electromagnetics Research Symposium (PIERS '13), pp. 139-143, Stockholm, Sweden, August 2013. 
[22] H. M. El Misilmani, M. Al-Husseini, K. Y. Kabalan, and A. El-Hajj, "Improved Vlasov antenna with curved cuts for high power microwaves," in Proceedings of the 11th International Conference on High Performance Computing and Simulation (HPCS '13), pp. 362-365, July 2013. 

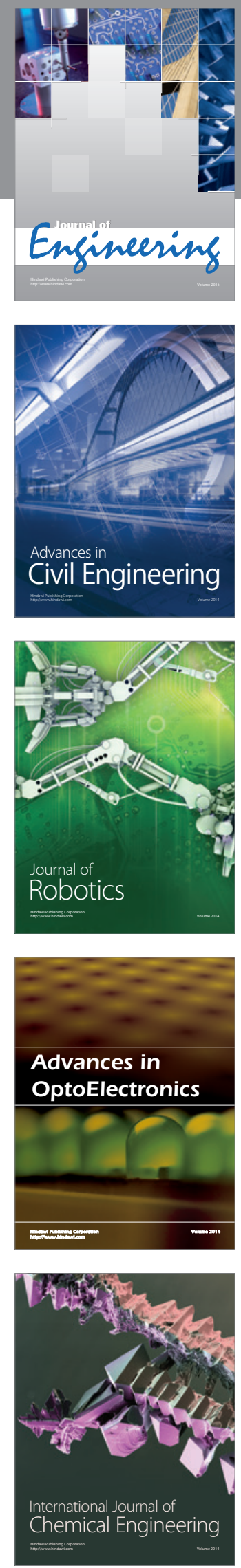

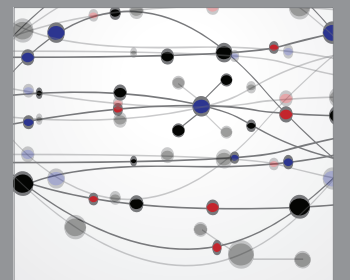

The Scientific World Journal
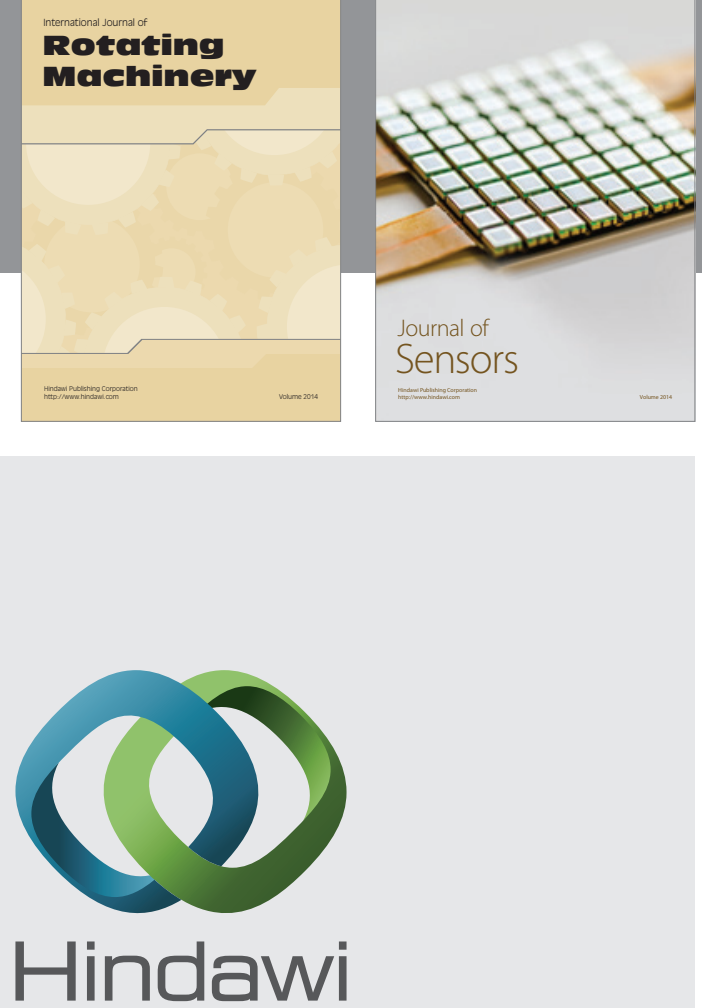

Submit your manuscripts at http://www.hindawi.com
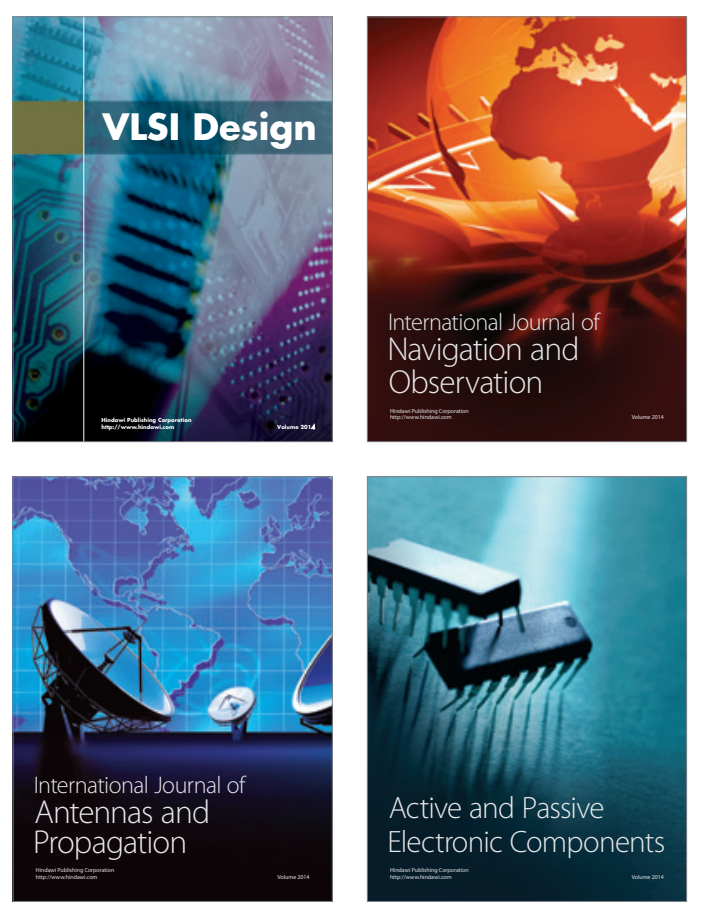
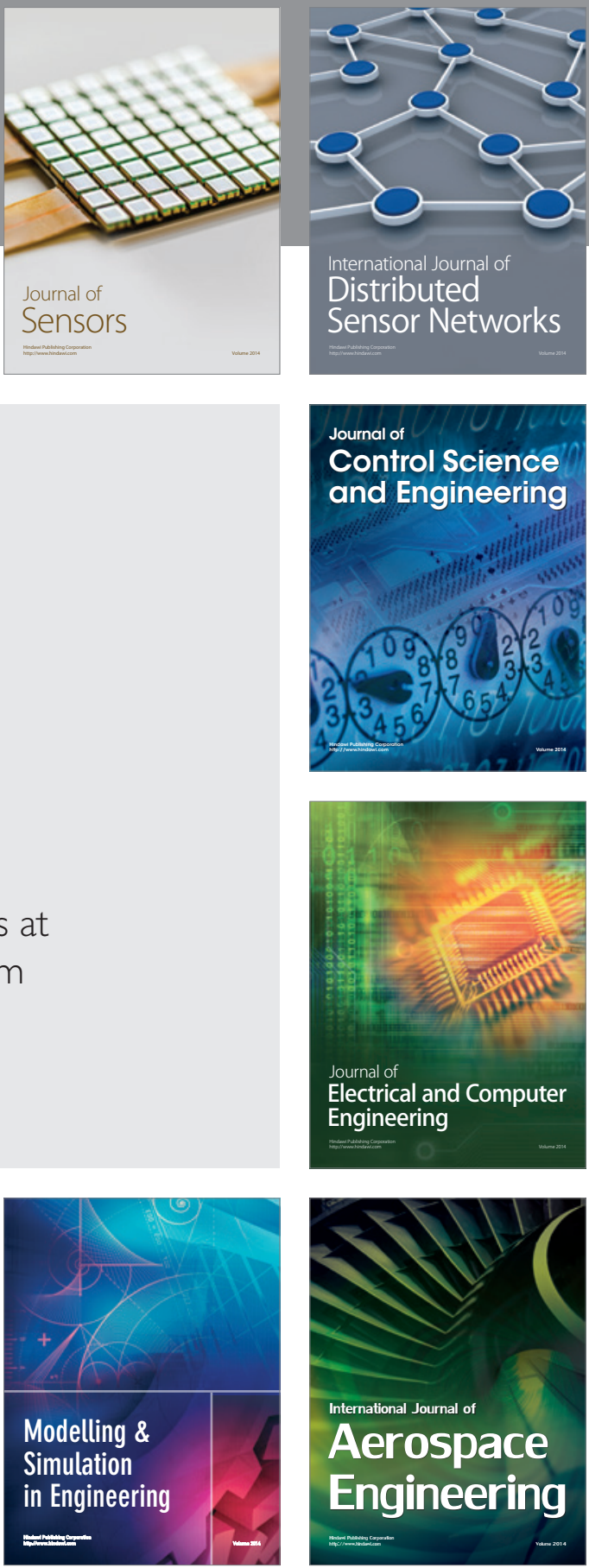

Journal of

Control Science

and Engineering
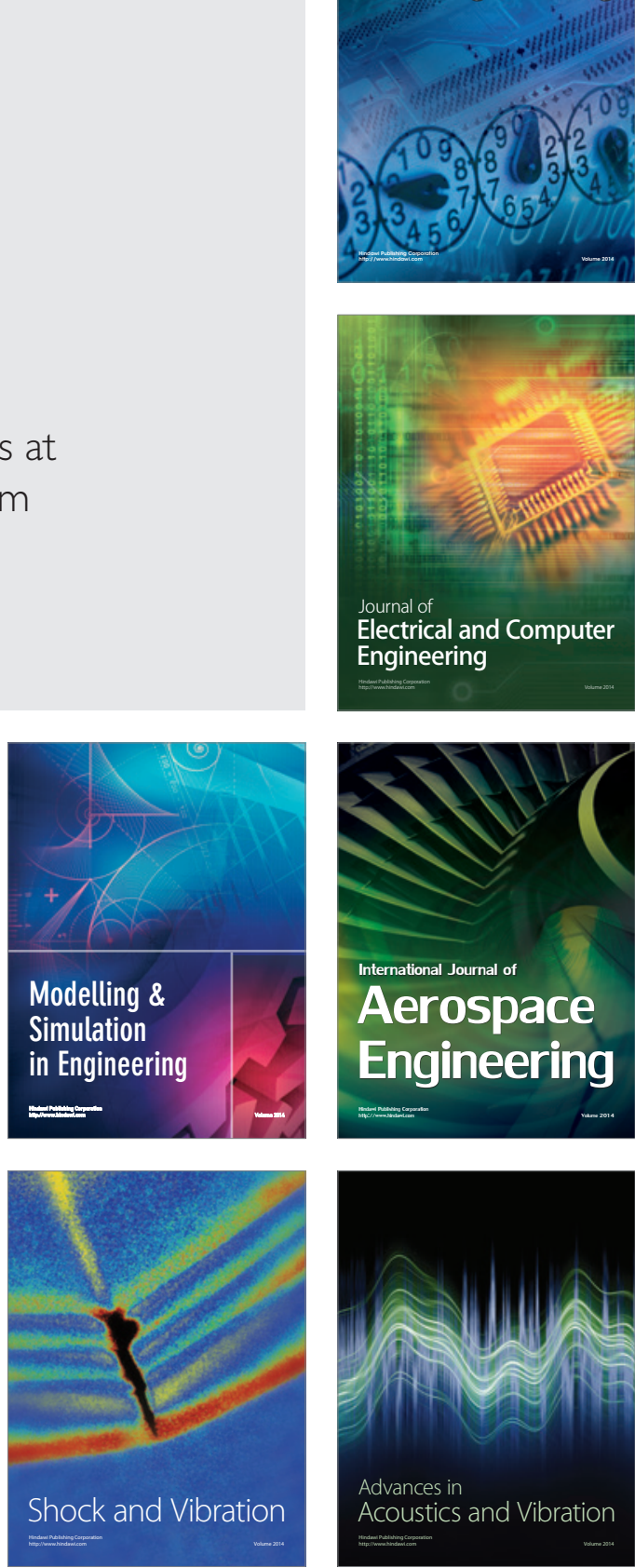